A N N A L E S Annales de Bretagne et des Pays de l'Ouest

Anjou. Maine. Poitou-Charente. Touraine

111-3 | 2004

Alcuin de York à Tours

\title{
Le dialogue de Franco et de Saxo
}

\section{Louis Holtz}

\section{OpenEdition \\ Journals}

\section{Édition électronique}

URL : http://journals.openedition.org/abpo/1229

DOI : $10.4000 /$ abpo. 1229

ISBN : 978-2-7535-1495-9

ISSN : 2108-6443

Éditeur

Presses universitaires de Rennes

Édition imprimée

Date de publication : 20 septembre 2004

Pagination : 135-145

ISBN : 978-2-7535-0053-2

ISSN : 0399-0826

\section{Référence électronique}

Louis Holtz, "Le dialogue de Franco et de Saxo ", Annales de Bretagne et des Pays de l'Ouest [En ligne], 111-3 | 2004, mis en ligne le 20 septembre 2006, consulté le 01 mai 2019. URL : http:// journals.openedition.org/abpo/1229; DOI : 10.4000/abpo.1229 


\section{III \\ Alcuin et les enjeux de l'écriture}





\title{
Le dialogue de Franco et de Saxo
}

\author{
Louis HOLTZ \\ Directeur honoraire de l'Institut de Recherche et d'Histoire des Textes (Paris)
}

La grammaire est pour Alcuin le premier des arts libéraux, non seulement par la place qu'elle occupe dans la liste des sept disciplines, ces degrés par lesquels on monte vers la sagesse et la connaissance de Dieu, mais parce qu'elle est à ses yeux une véritable propédeutique. Il faut passer obligatoirement par elle pour accéder à tout le reste, puisque c'est par elle que s'apprend la langue de l'Écriture sainte et de toute activité intellectuelle, le latin.

\section{Le De grammatica, œuvre de Tours}

Quand Alcuin entreprend la rédaction de son De grammatica, il a derrière lui à la fois le temps qu'il a passé comme professeur et aussi à la fin comme bibliothécaire à l'école épiscopale d'York, et les années vécues à la Cour de Charlemagne. Retiré dans son abbaye de Tours, l'une des plus grandes et des plus fameuses du royaume, son projet n'est pas du tout de se reposer (enfin!) de tant d'activités, mais plutôt de saisir l'occasion qui lui est donnée de parfaire son œuvre intellectuelle et spirituelle, loin de l'agitation mondaine au milieu de laquelle il vivait depuis qu'il avait rejoint la Cour du roi des Francs. En s'installant à Tours, d'itinérant qu'il était, de courtisan dépendant du bon vouloir du roi, il redevient sédentaire, maître enfin de lui-même, de son emploi du temps, de ses initiatives, et retrouve un milieu fait exclusivement de clercs, comme celui qui avait été le sien à York, un milieu dans lequel il peut partager entre la prière et l'activité intellectuelle le temps qu'il ne consacre pas à la direction du grand monastère. Détaché des obligations de Cour, il redevient spontanément un professeur.

Dans l'une des premières lettres envoyées de Tours, la lettre 121, écrite, selon Ernst Dümmler, en 796 après le 10 août, Alcuin trace à l'intention du roi, ce qui est désormais son programme :

"Quant à moi, votre Flaccus, pour répondre à vos encouragements et à votre affection à mon égard, sous le toit de saint Martin je désire servir aux uns le miel des saintes Écritures, enivrer les autres du vin vieux des antiques disciplines, commencer à en nourrir d'autres encore avec les fruits de la 
science subtile qu'est la grammaire, en éclairer plusieurs en leur montrant l'ordre des étoiles ${ }^{1} \ldots$...

Cette lettre écrite sur un ton lyrique est à la fois un programme et une sorte d'hymne au travail intellectuel. C'est aussi la lettre dans laquelle Alcuin demande à Charlemagne de l'autoriser à envoyer des coursiers à York pour en rapporter ceux de ses livres qui s'y trouvent encore, "les ouvrages (libelli), dit-il, que j'avais dans ma patrie, grâce à l'activité excellente et toute dévouée de celui qui fut mon maître ou même au prix de ma propre sueur, si peu que ce fût ${ }^{2}$ ". Il ne s'agit pas de n'importe quels livres, puisque ceux-ci sont qualifiés d'exquisitiores eruditionis scholasticae libelli, c'est à dire " particulièrement précieux par leur contenu scientifique fait pour l'école ". Il rêve d'avoir à nouveau à sa disposition les livres qu'il avait sous la main quand il enseignait à York. Si le roi a accédé à son désir, nous pouvons formuler l'hypothèse qu'il a alors reçu à Tours les livres du professeur qu'il était à York, qu'il a peut-être même récupéré des notes, ou des cours, ou encore des textes rédigés du temps où il enseignait dans sa patrie. Mais de toutes façons, même s'il n'a rien récupéré, ce qu'il nous dit là nous montre quelle était, lors de son installation à Tours, l'énergie intellectuelle qui était en lui pour opérer une sorte de retour aux sources de son propre savoir. Cela explique la productivité des années tourangelles d'Alcuin dans le domaine des arts libéraux et singulièrement, de la grammaire.

Le De grammatica, comme la Disputatio de vera philosophia qui la précède dans certains manuscrits, est né, à mon avis, dans ces années de réorganisation intellectuelle et d'enthousiasme, où il semblait à Alcuin qu'il recommençait sa vie de professeur et allait faire partager à un nouvel auditoire, celui des moines de Saint-Martin, les joies ineffables du savoir. On sait qu'en ce qui concerne les moines de Saint-Martin, il sera plutôt déçu de leur rusticité.

\section{Le genre du traité}

Dans quel genre le De grammatica se range-t-il aux yeux d'Alcuin luimême?

Au détour d'une phrase, devant les questions pressantes des élèves, le maître fait cette remarque : "votre curiosité est sans limite et c'est pourquoi vous voulez dépasser les limites d'un manuel ${ }^{3}$ ". Comme il est gênant de ne pas disposer d'un autre mot que manuel pour traduire le latin manualis libellus! C'est en réalité l'équivalent latin et même la traduction du grec egceiridion, c'est-à-dire le petit livre que l'on tient en mains ou encore qui

1. ALc. Ep. 121, p. 176.

2. Sed ex parte desunt mihi servulo vestro exquisitiores eruditionis scolasticae libelli, quos habui in patria per bonam et devotissimam magistri mei industriam vel etiam mei ipsius qualemcumque sudorem (ibid., p. 177).

3. Vestra curiositas modum non habet. Ideoque modum manualis libelli excedere vultis. (PL 101, 858 D). 
est à portée de main, donc un ouvrage dont on se sert tout le temps. L'adjectif manualis appliqué à libellus apparaît pour la première fois chez Taion de Saragosse, chez qui l'on trouve déjà la forme manuale ${ }^{4}$ au neutre substantivé, dans la préface à son épitomé de l'Histoire ecclésiastique d'Eusèbe. Taion explique qu'il veut réduire à un petit livre, capable de remémorer l'essentiel, l'immense ouvrage d'Eusèbe ${ }^{5}$. Tel est bien le sens de la qualification donnée par Alcuin à son ouvrage : c'est un bref opuscule, que les élèves auront à portée de main pour rafraîchir leur mémoire ${ }^{6}$, donc un opuscule à usage scolaire, censé résumer ce qu'il convient de savoir en grammaire.

\section{La mise en scène}

Ce petit traité est autant remarquable par sa mise en scène que par son contenu technique. Il porte l'empreinte de l'école du Palais, ne fût-ce que par la personnalité des deux personnages principaux qui s'y expriment.

Il nous donne à entendre la voix des élèves d'Alcuin, et d'une façon très vivante malgré la technicité du sujet. Ils sont représentés par deux d'entre eux, Saxo et Franco. Ces noms ne sont pas choisis au hasard. Ils symbolisent le double auditoire d'Alcuin, ils témoignent pour les élèves saxons et francs d'Alcuin. Ils rappellent qu'Alcuin a d'abord enseigné à des AngloSaxons avant d'avoir pour élèves les jeunes Francs de l'école du Palais. Saxo et Franco sont presque à égalité, dans l'âge comme dans la science, mais Saxo est quand même l'aîné, quoique d'une courte longueur : il a quinze ans, et Franco n'en a que quatorze. Mais c'est Franco, le plus jeune, qui est le demandeur.

" Il y avait à l'école de maître Albinus deux enfants, l'un Franco, l'autre Saxo, qui tout récemment se sont élancés à travers les buissons épineux de l'épaisse forêt grammaticale. C'est pourquoi ils ont décidé, pour exercer leur mémoire, d'extraire, en les récitant par demandes et par réponses, quelques règles qui caractérisent cette science des lettres. Mais c'est Franco qui, le premier prit la parole et dit à Saxo : Saxo, c'est moi qui vais poser les questions, et toi, y répondre ${ }^{7}$."

4. Le mot se rencontre peut-être d'abord chez Isidore, car il se trouve dans le recueil des gloses dites " isidoriennes " : Manuale, liber ad gerendum aptus qui enchiridion dici$\operatorname{tur}$ (PL 83, 1359, $\mathrm{n}^{\circ}$ 1019).

5. Liber manualis est le titre de l'ouvrage écrit vers 840 par Dhuoda pour son fils, cf. l'édition de P. Riche, B. de VREgille et C. Mondesert, Dhuoda, Manuel pour mon fils.

6. Alcuin emploie deux autres fois manualis libellus pour qualifier un de ses ouvrages : 1) dans la péroraison de son opuscule De virtutibus ac vitiis (PL 101638 B et cf. Alc. Ep. 305) datant des années 801/804 dédié à Wido, comte de la marche de Bretagne, un de ses élèves : ut habeas cotidie quasi manualem in conspectu tuo libellum, in quo possis te ipsum considerare, quid cavere, vel quid agere debeas. 2) dans le prologue aux Libri tres de fide sanctae Trinitatis, extraits du De Trinitate augustinien, une production sub specie manualis libelli (PL 101 12B), datant elle aussi de la période tourangelle, adressée à Charlemagne pour être remise au clergé de l'Empire au plaid d'Aix-la-Chapelle de 802.

7. Fuerunt in schola Albini magistri duo pueri, unus Franco, alter Saxo, qui nuperrime spineta grammaticae densitatis irruperunt. Quapropter placuit illis paucas litteralis 
La première chose à remarquer, c'est qu'Alcuin lui-même se met en scène sous le nom d'Albinus : c'est sa voix à lui que nous entendons au deuxième degré quand le maître intervient. Mais Saxo est par rapport à Franco dans la situation d'Alcuin par rapport à Charlemagne, qui est lui aussi le demandeur, comme on le voit dans les autres dialogues, le De rhetorica et le De dialectica; en effet, Saxo en sait un peu plus que son interlocuteur, car il connaît et il fournit les réponses. Mais ce qui est primordial, c'est quand même l'initiative de poser les questions et ce rôle est donné à Franco, qui contrôle ainsi, apparemment du moins, le déroulement de la récitation et par ses questions, qui prennent une forme variée et parfois provocante, révèle son appétit de savoir et garantit la dynamique de la récitation. On pense à cette réflexion qu'Alcuin prête à Charlemagne dans le dialogue De rethorica et virtutibus et qu'il lui rappelle dans deux lettres : interrogare sapienter est docere, " interroger avec sagesse, c'est enseigner et s'il y en a un qui interroge, un autre qui enseigne, la façon de penser chez tous les deux procède de la même source, la sagesse ${ }^{8}$ ". Alcuin a tout fait pour donner de la vie à cette sorte de jeu, qualifié par les acteurs euxmêmes de lusus, et il y est parvenu en donnant à chacun des deux partenaires une personnalité, ce qui fait de l'opuscule d'Alcuin un texte très différent des grammaires dialoguées entre le maître et l'élève que les écoles du haut Moyen Âge ont multipliées sur le modèle de l'Ars minor de Donat.

\section{La présence du professeur}

Cependant les deux élèves ne sont pas livrés à eux-mêmes. Ils récitent en présence de leur professeur. C'est la condition mise par Saxo.

" D'accord, répondit Saxo, à condition toutefois que s'il est nécessaire de poser une question plus approfondie ou de recourir à la discipline philosophique, il soit permis d'interroger notre professeur ${ }^{9}$."

Et donc le professeur, Alcuin lui-même, lance le dialogue en posant la question "par quoi pensez-vous qu'il faut commencer?".

Les élèves s'adressent au maître d'une seule voix ${ }^{10}$. Quand celui-ci leur demande par quoi il faut, à leur avis, commencer la récitation, Franco et Saxo répondent en chœur, "Par quoi commencer? par le chapitre de la lettre bien sûr. ". Certes ils sont fondés à commencer par l'étude de la lettre s'il est vrai que grammatica a pour équivalent en latin scientia litteralis, traduction empruntée à Isidore qu'approuve le maître ${ }^{11}$. Mais cette considé-

scientiae regulas memoriae causa per interrogationes et responsiones excerpere. At prior illorum Franco dixit Saxoni : Eia, Saxo, me interrogante responde. Cette introduction nous ramène dans un passé récent mais révolu.

8. PL 101, 939 A; cf. Alc. Ep. 136, p. 205; Alc. Ep. 308, p. 471.

9. Ad haec Saxo respondit : faciam, ita tamen, ut si quid altius sit interrogandum, vel ex philosophica disciplina proferendum, liceat magistrum interrogare. (PL 101, $854 \mathrm{~B}$ )

10. Dans le texte d'Alcuin le sigle des intervenants n'est alors ni Fr[anco], ni Saxo mais Dis[cipuli].

11. Cf. PL 101, 857 D. 
ration étymologique ${ }^{12}$ ne règle pas tout. Car la lettre, n'est pas autre chose que la transcription par l'écrit d'un élément qui existe avant elle, le son vocal $^{13}$.

« Non, dit le maître, c'est par la voix, puisque la lettre a été inventée pour la fixer, que doit commencer votre discussion (collocutio, disputatio). Ou plutôt, c'est sur quels éléments est fondée une discussion qu'il faut d'abord s'interroger ${ }^{14}$."

La présence du professeur a donc pour objet de faire prendre aux élèves du recul tout en les mettant sur la bonne voie, qui est celle de la dialectique. Cette indication liminaire, dans laquelle le maître recourt à Aristote par l'intermédiaire du commentaire au De interpretatione de Boèce, ne va pas transformer la récitation des deux élèves en un dialogue socratique, loin s'en faut. Mais elle montre que pour Alcuin la grammaire, si humbles soient ses enseignements, - et ils restent humbles dans le De grammatican'est jamais une fin en soi.

Le maître n'intervient vraiment qu'au début du dialogue, c'est-à-dire dans la première partie de l'ouvrage, qui, par sa disproportion avec ce qui suit, donne l'impression d'être seulement une introduction à cet opuscule. En effet, si nous nous référons au canevas usuel des manuels de grammaire, l'essentiel, la partie centrale, est représentée toujours au haut moyen âge par l'étude des parties du discours. Du reste, beaucoup de traités grammaticaux se réduisent à cela. Le De grammatica d'Alcuin ne fait pas exception : sur les 47 colonnes du tome 101 de la Patrologie, les parties du discours en occupent 42, étudiées dans l'ordre qui est celui de Donat : cela correspond au livre II de l'Ars maior du grammairien antique, tandis que les cinq premières colonnes de Migne, cette sorte d'introduction, reposent sur le premier livre de l'Ars maior. En fait, le maître est là pour surveiller que la discussion prend un bon départ, et une fois qu'elle est lancée, il s'éloigne discrètement. Sur ses quatorze ${ }^{15}$ interventions dans ce petit traité, onze se situent dans la brève partie introductive, et trois seulement dans l'étude des parties du discours.

Mais il ne nous est pas dit que le maître s'éloigne, cela est simplement suggéré par la plus grande liberté que prennent peu à peu les élèves, non pas vis à vis du contenu grammatical, mais entre eux, dans leur façon de se parler. Ils finissent même par se chamailler. Saxo n'en peut plus à force de réciter tout ce qu'il sait ${ }^{16}$ et traite Franco, qui en veut trop, d'avidus. Mais celui-ci continue malgré tout à le presser de questions, en lui repro-

12. Cette étymologie est indirectement empruntée à Isidore de Séville, Etymologiarum..., $\mathrm{I}, 5,1$.

13. La remarque du maître a pour effet de corriger l'exposé isidorien, qui commence par l'étude des lettres en négligeant le son vocal.

14. A voce, cuius causa litterae sunt inventae, inchoandam disputationem constat : vel magis primo omnium interrogandum est quibus modis constet disputatio. (PL 101, $854 \mathrm{C}$ ).

15. PL 101, 854 C (2 fois), 854 D (2 fois), 855 A, 855 B, 857 BC, 857 D (2 fois), 858 A/D, 858 D-859 A, 859 BC, 874 A, 874 AB.

16. Cf. PL 101, 867 D. 
chant d'être, lui, invidus (jaloux), de vouloir garder pour lui tout ce qu'il sait ${ }^{17}$. La récitation devient ainsi dialogue sur un canevas connu, un dialogue dynamisé par le débit de l'un et les questions pressantes de l'autre. Le jeu se prolonge et risque de se terminer, comme il est suggéré, à la lumière des lanternes ${ }^{18}$. Finalement, les deux élèves conviennent de faire quelques pauses, mais seulement après le De verbo, après le De adverbio. Et les voilà maintenant qui parlent du maître par allusion ou à la troisième personne, ce qui est à la limite de l'irrespect, mais également le signe de son absence. Franco dit que les punaises qui sont dans la maison du maître lui ont rempli les oreilles de leurs petits problèmes ${ }^{19}$. Ou encore, après un échange de réflexions aigres douces Saxo prévient : "Fais gaffe! celui qui d'habitude donne le fouet pourrait entendre ${ }^{20}$. " Le maître n'est donc pas loin, mais il n'assiste plus en tiers à cette à la discussion.

\section{Les interventions du maître : l'aspect philosophique}

Ses interventions ont beau être en nombre limité, elles n'en méritent pas moins d'être relevées soigneusement, car mieux encore que les propos des élèves, elles sont représentatives des conceptions d'Alcuin luimême, et des nouveautés qu'il introduit dans la vulgate grammaticale, ne fût ce que par la synthèse qu'il opère entre des sources diverses.

Le maître, Alcuin donc, intervient de deux façons, en référence soit à la philosophie soit à la grammaire. En référence à la philosophie, nous comptons trois interventions. Elles sont toutes les trois inspirées du commentaire au De interpretatione de Boèce. J'en ai déjà mentionné une, savoir, se demander sur quoi repose un dialogue. Les deux autres sont beaucoup plus significatives, parce qu'elles engagent l'avenir même de la réflexion linguistique et revêtent comme un caractère prophétique. Il s'agit de l'introduction dans la grammaire, pour la première fois en Occident, des définitions aristotéliciennes du nom et du verbe qu'Alcuin connaît à travers le même Boèce.

Certes, ces deux définitions aristotéliciennes se rencontrent chez Cassiodore, lecteur de l'œuvre de Boèce, son parent, et chez Isidore, qui les a purement et simplement recopiées à partir du livre II des Institutions de son devancier, mais chez les deux auteurs, ces définitions figurent non pas dans la grammaire, mais là où on les attend, dans le chapitre consacré à la dialectique. Il s'agit de savoir, chez Boèce comme chez eux, à quelle condition une proposition est vraie ou fausse. Chez Alcuin, il en va autrement : nous trouvons les définitions aristotéliciennes (boéciennes) du nom et du verbe deux fois, dans le De dialectica mais aussi dans le De grammatica. Les deux emprunts diffèrent quelque peu.

17. Même réaction de Charlemagne vis à vis d'Alcuin dans le De rhetorica: An invides ut hoc sciam? (PL 101, 939 A).

18. PL 101, 865 C.

19. PL 101, 870 D.

20. PL 101, 881 CD. 
Pour faire bref, je dirais qu'avec l'introduction dans le De grammatica d'Alcuin de la définition aristotélicienne du nom, nous assistons à la grammaticalisation de notions dialectiques, ou à la récupération par la grammaire d'éléments mis en œuvre jusque là uniquement par les dialecticiens. En mettant côte à côte la définition de Boèce et l'emprunt que lui fait Alcuin dans ce traité ${ }^{21}$, on note tout de suite qu'un membre de phrase important dans la définition aristotélicienne du nom ne figure plus chez Alcuin, savoir cuius nulla pars significativa est separata, qui dans la pensée d'Aristote oppose le nom ou le verbe à l'énoncé complet, l'oratio, dont certaines des parties, précisément le nom ou le verbe, quand elles sont séparées de la phrase, sont porteuses, elles, de sens par elles-mêmes. Alcuin dans son $D e$ grammatica n'a donc pas voulu s'engager sur une voie qui l'aurait mené à l'étude de la syntaxe, dont l'idée n'est directement présente dans le traité, sinon par la définition du terme oratio, empruntée à Priscien ${ }^{22}$. De plus, de la définition du De grammatica disparaît toute référence aux notions de vrai et de faux. Le contexte philosophique lui-même s'évanouit, il n'est plus même question de Socrate ou de Caton.

La même définition boécienne recueillie par Alcuin dans son De dialectica n'omet pas le membre de phrase cuius nulla pars... separata et baigne dans un environnement philosophique (Socrates). Toutefois la référence aux notions de vrai et de faux n'est introduite que plus loin, à propos de l'enunciatio (974 CD). Si on compare les deux emprunts alcuiniens à Boèce pour la même définition on observe (sous réserve d'une vérification critique de l'établissement du texte ici et là) que l'emprunt du De gram-

21. Boethius, In librum De interpretatione Aristotelis, PL 64, 306 AB sqq. : Nomen est vox significativa secundum placitum, sine tempore, cuius nulla pars significativa est separata, definitum aliquid significans, cum est aut non est iuncta faciens enuntiationem. (ibid. 306 $B$ ). In casibus vero, quamvis addas est aut non est, nulla inde affirmatio neque negatio fit. Neque enim in hos ulla veritas aut falsitas cadit. Si quis enim dicat Socratis est, Socratis non est vel Catonis est, Catonis non est, quid sit vel non sit non apponens, cum simplici casu est aut non est iungens, imperfectam faciet orationem a falsitate ac veritate disiunctam. (306 A)//Alcuin, De grammatica, PL 101, 859 BC : MAG. Nomen est vox significativa secundum placitum, sine tempore, definitum aliquid significans in nominativo cum est aut non est, ut homo est, homo non est. In [aliis] casibus licet addas est aut non est, nihil tamen certum significat, si non apponas quid sit vel non sit, ut hominis est, hominis non es+t. Secundum placitum, id est compositionem singularum gentium sunt nomina composita, ut quod latine dicis aurum, hoc graece $\chi \rho v \sigma o \sigma$ ? dicitur. Una est substantia, sed diversa nomina.

22. Priscien, Institutions grammaticales, Grammatici Latini II, 53, 28. Étrangement Alcuin dans le De grammatica emprunte la définition d'oratio à Priscien sous une forme inexacte, alors que dans le De dialectica il donne la même définition (empruntée là encore à Priscien, non à Boèce) sous une forme non altérée. On comparera Oratio est ordinatio dictionum congrua, sententiam perfectam demonstrans, Priscien, "Institutions grammaticales " Grammatici Latini II, 53, 28 (mais certains mss de Priscien portent congruam... perfectamque)//(oratio est) congrua partium ordinatio, perfectam sententiam demonstrans, Alcuin, De dialectica, PL 101, 974 C//Oratio est ordinatio dictionum congruam sententiam perfectamque demonstrans; Alcuin, De grammatica, PL 101, 858 A. C'est cette définition altérée qu'on lit à la suite d'Alcuin chez plusieurs grammairiens du IX ${ }^{e}$ siècle. Cf. A. LuHTALA, "Excerpta da Prisciano... », p. 332-335 et n. 27. 
matica est beaucoup plus fidèle ${ }^{23}$ à la rédaction littérale de Boèce, comme s'il était antérieur.

Mais dans les deux emprunts la notion de cas est grammaticalisée. Aristote comme Boèce, opposent la forme brute du nom à la forme des cas que reçoit le nom et donc Boèce ne prononce pas le mot nominativus ${ }^{24}$. Pour Alcuin le nominatif, quelles que soient ses propriétés, est un cas comme les autres, parce qu'il a une morphologie à lui, et donc l'addition des mots in nominativo est tout à fait significative, même si elle ne modifie pas le fond du problème.

\section{Les interventions du maître : l'aspect grammatical}

Voyons maintenant les interventions du maître en référence à la grammaire. Le maître se réserve certains apports qui marquent un élargissement de la culture grammaticale traditionnelle.

Ainsi nous l'avons déjà vu rétablir en tête de la récitation des élèves le chapitre De voce. Un peu plus loin, sans nommer Isidore, mais nous reconnaissons bien la dette, c'est le maître qui énonce que la grammaire comporte de nombreux aspects (species), qui ne se trouvent pas dans les artes grammaticae, c'est-à-dire outre les chapitres contenus dans la grammaire de Donat, et qui sont bien connus, son vocal, lettre, syllabe, pieds, accents, signes de ponctuation, huit parties du discours, barbarisme, solécisme, défauts du discours, métaplasme, figures, tropes, il faut ajouter avec Isidore les signes graphiques (notas), l'orthographe, l'analogie, l'étymologie, les glossae ou explications de mots, les différences, la prose, les mètres, les récits fictifs (fabulas) ou de choses vécues (historias). La liste d'Alcuin reprend celle d'Isidore mais les deux auteurs ont une manière différente de faire l'addition; la liste d'Alcuin est même plus longue puisqu'il ajoute les dictiones, les orationes, les deffinitiones ${ }^{25}$, ce qui, pour ce dernier concept, est une innovation qui lui est propre et dont la présence relève aussi de la grammaticalisation d'une notion traitée jusque là uniquement par les philosophes et notamment par Marius Victorinus.

C'est le maître encore qui ajoute deux notations importantes empruntées l'une et l'autre à Diomède, contemporain et utilisateur de Donat. Un

23. On comparera : cum est aut non est (Boèce, PL 64, 306 A, Alcuin PL 101, 859 B)//cum est et non est (Alcuin, PL 101, 973 A); quamvis addas est aut non est (Boèce, ibid.), licet addas est aut non est (Alcuin, PL 101, $859 \mathrm{~B}$ )//nisi additis (Alcuin); quid sit vel non sit (Boèce, Alcuin)//aliis partibus orationis (Alcuin).

24. Faute d'une édition critique, je ne sais comment interpréter la leçon que donnent certains mss du De grammatica en PL 101, 859 B : in casibus (comme Boèce PL 64, $306 \mathrm{~A}$ : in casibus vero) alors que les autres écrivent in aliis casibus, comme par prétérition, étant donné que nominativus va être mentionné un peu plus loin dans la phrase : correction d'Alcuin qui après avoir adopté le texte de Boèce l'a complété au cours d'une relecture? Le De dialectica porte in aliis vero casibus (mixture du texte de Boèce et de celui du De grammatica révisé?).

25. Sur l'addition des deffinitiones, cf. A. LuHTALA, "Excerpta da Prisciano... ", p. 332335 et n. 27. 
manuscrit de ce grammairien avait été offert à Charlemagne vers 780 par le moine Adam de Masevaux, et c'était donc un auteur redécouvert dont le texte a été lu par les érudits de la Cour. On a montré ${ }^{26}$ par exemple comment l'ars de Diomède avait également influencé Pierre de Pise. Alcuin emprunte à Diomède d'une part l'énumération des fondements de la grammaire :

« La grammaire repose sur quatre éléments, le donné naturel de la langue (natura), la règle logique ou plutôt analogique (ratione), le modèle fourni par les auteurs (auctoritate) et l'usage (consuetudine) ${ }^{27}$."

La formulation alcuinienne est une sorte de compromis entre celle de Diomède et celle d'Audax pour cette définition qui remonte à Varron.

La seconde notation empruntée par le maître lui-même à Diomède est celle de la composition des éléments du langage depuis la lettre isolée jusqu'à la phrase porteuse d'une pensée. Diomède disait, là encore en reflétant une formule varronienne :

"Les premiers pas de la grammaire sortent des sons élémentaires, les sons élémentaires sont représentés par les lettres, les lettres se réunissent en syllabes, par les syllabes se réalise le mot, les mots se réunissent en parties du discours, les parties du discours trouvent leur achèvement dans le $\operatorname{discours}^{28}$."

La même pensée est formulée par Alcuin, mais en sens inverse :

"La lettre est insécable parce que nous divisons les phrases en parties du discours, les parties du discours en syllabes, les syllabes en lettres. Quant aux lettres, on ne peut les diviser ${ }^{29}$."

Je voudrais ici insister sur le génie d'Alcuin qui, dans sa lecture de Diomède, a tout de suite remarqué l'importance de ces deux enseignements qui remontent à Varron et les a placés dans cette partie introductive du traité. Ils ont en commun la perception de ce qui dans le langage est dynamique.

La récitation des élèves doit beaucoup, comme nous allons le voir, à Priscien. Mais le maître se réserve d'exposer une pensée qu'il a rencontrée

26. Cf. A. LuHTALA, ibid. p. 330 sqq.

27. Quae (= grammatica) constat natura, ratione, auctoritate, consuetudine (PL 101, 857 D). Seuls Charisius (62, 14 B.) et Diomède (Grammatici Latini I, 439, 16) mentionnent quatre principes (avec natura en tête) : natura, analogia consuetudine auctoritate (analogia étant comme l'explique Quintilien (Inst. orat., I, 6, 1), l'application d'un principe rationnel, ratio, d'une règle). Le reste de la tradition grammaticale n'en mentionne que trois parce que natura (le donné naturel de chaque langue) va de soi et qu'on ne peut le modifier. Ces trois principes se présentent dans l'ordre : ratione, auctoritate, consuetudine chez Audax, Grammatici Latini VII, 322,22 (= Victorinus Grammatici Latini VI, 189,3), S. Augustin Ars breviata 7,4 (W.). L'énumération d'Alcuin, qui remet en faveur le terme natura, suppose une lecture de Diomède (Charisius étant moins probable) et fait la synthèse entre les deux traditions.

28. Diomède, Grammatici Latini I, 426, 32 cf. G FunaIol, Grammaticae Romanae fragmenta, Varron, fr. 237.

29. PL 101, 855 A. 
chez le grammairien de Constantinople et qui n'a pas de correspondance chez les autres grammairiens latins, la comparaison entre le couple voyelles/consonnes et le couple âme/corps. Les voyelles donnent vie aux consonnes, comme l'âme met en mouvement le corps. Voilà encore une notation dynamique.

Enfin Alcuin, conformément à une division des disciplines héritée de l'Antiquité tardive, met à part de la grammaire ce qui concerne la métrique, et c'est pourquoi le maître prend à son compte l'exposé concernant les syllabes communes ${ }^{30}$, qui concerne la valeur des syllabes dans le vers. Mais, c'est aussi parce qu'il a l'intention d'écrire pour Franco et Saxo un traité de métrique ${ }^{31}$. Cette promesse, nous le savons, ne sera pas tenue.

Les interventions du maître, tranchent, par leurs orientations, sur le contenu des propos que tiennent les deux élèves. Je voudrais pour finir m'arrêter seulement sur quelques aspects de leur dialogue.

\section{Quelques aspects du dialogue des élèves}

Dans le cadre formé par les huit parties du discours, étudiées dans l'ordre et souvent avec les mots de Donat, sont injectées toutes sortes de notions fondamentales, qui témoignent de la part de l'auteur d'une immense culture grammaticale : les sources en sont révélatrices. D'abord le Priscien des seize premiers livres des Institutions grammaticales, qui dans la récitation faite par Saxo submerge d'un bout à l'autre l'enseignement de Donat. Il arrive qu'un paragraphe tout entier entremêle l'enseignement des deux grammairiens antiques. Ainsi le paragraphe consacré aux différentes catégories de noms appellatifs ${ }^{32}$. Le canevas de l'énumération est celui de l'Ars maior $(615,1)$ : species multae sunt : alia corporalia alia incorporalia alia etc. Mais parmi les catégories donatiennes se glissent des catégories propres à Priscien. Et ce même schéma se renouvelle à chaque instant.

Il est particulièrement intéressant d'observer comment sont traités les cas où l'enseignement des deux grammairiens diverge. Par exemple pour les accidents du verbe, qui sont énumérés selon la liste de Priscien ${ }^{33}$, la curiosité de Franco est éveillée du fait que Donat ne traite pas de l'aspect (species). Mais Saxo lui montre sans difficulté que ce que Priscien appelle

30. PL $101,857 \mathrm{~A} / \mathrm{C}$.

31. PL 101, $857 \mathrm{C}$ : plenius haec, vita comite, in metrica ratione vobis, filii, monstravero. On notera l'expression vita comite, empruntée à saint Jérôme, qui dans sa version de la Genèse (XVIII, 10) met ces mots à deux reprises dans la bouche des trois visiteurs d'Abraham sous le chêne de Membré. L'expression se rencontre après lui chez Isidore et surtout chez les écrivains anglais, Bède, Aldhelm, Boniface. Alcuin l'a déjà employée dans le Dialogus de vera philosophia ou "Introduction aux disciplines" (PL 101, 853 C/D) pour une autre promesse conditionnelle faite à ses élèves.

32. PL 101, $860 \mathrm{~A} / \mathrm{C}$.

33. Priscien, Grammatici Latini II, 368, 16. Curieusement, Alcuin ne mentionne pas la coniugatio dans la liste des accidents du verbe. 
species, Donat en traite dans l'étude de la forma ${ }^{34}$; il lui montre pareillement ${ }^{35}$ que ce que Priscien nomme catégories (species) de conjonctions Donat l'appelle potestas (pouvoir) : et si Priscien énumère plus de catégories que Donat, c'est parce que celui-ci se borne aux principales, qui englobent déjà celles qu'ajoute Priscien. La différence entre les deux grammairiens a donc tendance à être minimisée. Franco s'inquiète de ce que Donat (magister noster Donatus) ne donne sur les verbes ou les mots défectifs que des explications peu claires et trop rapides. Il s'attire cette réponse de son partenaire : "Je ne suis pas débiteur à ton égard des faits de langue irrationnels, mais des faits de langue rationnels, et qui sont soumis à des règles ${ }^{36}$. "Saxo va quand même essayer de relever le défi en ébauchant une théorie des termes défectifs qui est un résumé bien conduit de Priscien ${ }^{37}$ : là encore, Priscien est appelé à compléter ou à expliciter Donat, au-delà duquel, semble-t-il, Franco n'est pas encore allé bien loin, alors que Saxo a retenu le contenu, lexicalement très documenté, des «Institutions grammaticales ". À travers les propos de Saxo, Franco découvre Priscien.

Il y a deux cas où les doctrines des deux grammairiens sont inconciliables, celui des formes du gérondif (ad amandum, etc.), qui sont des formes verbales pour Donat, nominales pour Priscien ${ }^{38}$, et celui de mots tels que qualis, talis $^{39}$ etc. classés parmi les pronoms chez Donat et parmi les noms chez Priscien. Ici Saxo tend à abonder dans le sens de Priscien, ce qui revient à dire aussi que c'est la position d'Alcuin. Ce mélange de Priscien et de Donat renvoie au grand poème sur York où Alcuin décrit la bibliothèque dont il avait aussi la charge dans les dernières années de ses fonctions dans son pays. Énumérant les livres de grammaire qui s'y trouvaient, il nomme dans le second hémistiche d'un vers ${ }^{40}$, Donatus Priscianusve, les deux auteurs antiques principaux, déjà liés dans sa pensée de façon indissociable. Il est vraisemblable que depuis ce temps l'admiration d'Alcuin pour Priscien n'a fait que croître : pour les matières communes aux deux grammairiens (Ars maior I et II d'une part, "Institutions

34. PL 101, 877 D. Même cas pour les accidents du nom : Saxo montre que Priscien groupe sous l'accident species ce que Donat traite sous les accidents qualitas et comparatio (PL 101, $859 \mathrm{C})$.

35. PL 101, 895 B. Il s'agit des verbes fréquentatis, inchoatifs, méditatifs, etc.

36. Non sum tibi debitor irrationabilium, sed rationabilium, et eorum quae regulis subiaceant. L'expression regulis subiaceant fait écho à la fameuse déclaration de saint Grégoire dans la préface-dédicace des Moralia in Iob. Que les défectifs ne soient pas soumis à des règles, c'est ce que note Pompée, Grammatici Latini V, 238, 36 : Nemo dat regulas in verbis defectivis.

37. Cf. Priscien, Grammatici Latini II, 369, 18-373, 8.

38. PL 101, 877 C, 880 B.

39. PL 101, 873 C. Ici Saxo tend à abonder dans le sens de Priscien, qu'il qualifie de latinae eloquentiae decus. Cet éloge est de même genre que celui que nous lisons dans la lettre 52 à Angilbert (vers 796-799 selon Dümmler) : Priscianus veracissimus artis grammaticae doctor (MGH, Ep. t. 4, lettre 162, p. 262, 21). J'ai montré ailleurs combien cette réponse à une consultation grammaticale supposait chez Alcuin une fine connaissance des Institutions grammaticales de Priscien.

40. Alcuin, The Bishops, Kings and Saints of York, Carmen 1, v. 1555. 
grammaticales " I-XVI d'autre part), Donat reste pour Alcuin l'auteur de base, mais ce qui compte surtout pour lui, c'est que cet enseignement élémentaire ne peut se passer d'être enrichi par celui de Priscien.

\section{Le travail d'un pédagogue}

En fait ce traité est comme le résumé de la culture grammaticale d'Alcuin, en ce qu'il est écrit non pas avec des livres que l'auteur démarquerait, mais avec la mémoire de lectures immenses et de première main, qui englobent une grande partie des textes publiés dans les Grammatici latini de Keil. Priscien et Donat représentent peut-être 80 \% des sources, mais à titre complémentaire nous trouvons, outre Diomède, Pompée, Phocas, Isidore, le livre I des Explanationes, Audax, et même Bède et son De arte metrica. Mais quand on cherche les sources de cet opuscule, on les relève très rarement sous un aspect littéral.

Je dirais en conclusion que le De grammatica est avant tout l'ouvrage d'un pédagogue réaliste, conscient des besoins du temps et du niveau des élèves, soucieux de consolider, d'enrichir et même de renouveler les bases traditionnelles de l'enseignement de la langue, mais aussi de ménager des étapes. Donat première étape niveau de Franco, Donat complété par Priscien, deuxième étape, niveau de Saxo; et précisément Saxo, dans le De grammatica, aide Franco à passer à la seconde étape. Alcuin n'a certainement pas dédaigné l'apport des livres 17 et 18 des Institutions grammaticales de Priscien et s'est attaché lui-même à maîtriser le contenu d'une discipline nouvelle dans l'histoire de l'enseignement de la grammaire, en tirant des deux derniers livres de Priscien ces Excerptiones super Priscianum ${ }^{41}$ qui portent sa marque, mais il était hors de question à son époque de ménager à la toute nouvelle syntaxe une place dans l'enseignement, et d'en faire la matière d'une troisième étape. Telle est aussi par leur silence la position de Clément et celle du meilleur élève d'Alcuin à Tours même, Raban Maur, qui l'un et l'autre dans leurs traités ne connaissent comme ouvrage grammatical d'Alcuin que le De grammatica et semblent ignorer totalement les livres 17 et 18 des Institutions grammaticales.

41. Cf. L. Holtz, « Priscien dans la pédagogie... », p. 289-326. 


\section{RESUME}

L'installation d'Alcuin à Tours est pour lui une nouvelle étape. Son programme est ambitieux, quand libéré de la Cour il se retrouve professeur. Mais il a maintenant derrière lui à la fois son enseignement à York et à la Cour, ce que symbolise les noms de Franco et de Saxo. Ce " liber manualis " est autant remarquable par sa mise en scène que par son contenu. Saxo, à peine plus âgé en sait plus, mais c'est Franco qui interroge, comme Charlemagne dans le De rhetorica. Les interventions du maître tirent vers le haut et vers le neuf cette récitation où les Institutions de Priscien sont coulées dans le moule de Donat, Ars maior I et II. C'est l'œuvre de quelqu'un qui a longuement assimilé les grammairiens antiques, car les sources ne sont jamais littérales, mais aussi d'un pédagogue prudent et soucieux de ne pas brûler les étapes.

\section{ABSTRACT}

Alcuin's arrival at Tours marked a new stage in his life. His programme was ambitious and, when free from the Court, he returned to teaching. But now he had his experience both in York and at the Court under his belt, as symbolized by the names of Franco and Saxo. This liber manualis is as remarkable for its setting as for its content. Saxo, who is barely older, knows more, but Franco poses the questions, like Charlemagne in the De rhetorica. The master's comments elevate and freshen this recitation, in which the Institutions of Priscian are cast in the mould of the Ars maior I and II of Donatus. It is the work of someone long acquainted with the ancient grammarians, because the sources are never cited literally, but it also reflects the prudent pedagogue who proceeds slowly and carefully. 
